\title{
Impact of Social Distancing Due to Coronavirus Disease 2019 on the Changes in Glycosylated Hemoglobin Level in People with Type 2 Diabetes Mellitus (Diabetes Metab J 2021;45:109-14)
}

\author{
Sung-Don Park ${ }^{1}$, Sung-Woo Kim² , Jun Sung Moon 3 , Jae-Han Jeon ${ }^{4}$, Mi Kyung Kim5, Keun-Gyu Park ${ }^{1}$ \\ ${ }^{1}$ Department of Internal Medicine, Kyungpook National University Hospital, School of Medicine, Kyungpook National University, Daegu, \\ ${ }^{2}$ Department of Internal Medicine, Daegu Catholic University Hospital, Daegu Catholic University School of Medicine, Daegu, \\ ${ }^{3}$ Department of Internal Medicine, Yeungnam University Hospital, Yeungnam University College of Medicine, Daegu, \\ ${ }^{4}$ Department of Internal Medicine, Kyungpook National University Chilgok Hospital, School of Medicine, Kyungpook National University, Daegu, \\ ${ }^{5}$ Department of Internal Medicine, Keimyung University Dongsan Hospital, Keimyung University School of Medicine, Daegu, Korea
}

We would like to thank Dr. Noh for your interest in and comments regarding our study, entitled "Impact of social distancing due to coronavirus disease 2019 on the changes in glycosylated hemoglobin level in people with type 2 diabetes mellitus," which was published in Diabetes \& Metabolism Journal [1].

At the beginning of the coronavirus disease 2019 (COVID-19) outbreak in Daegu, the number of patients contracting COVID-19 has been skyrocketing [2], and strict social distancing, which is currently level-3 [3], was implemented to prevent transmission of infection [4]. Social distancing affects peoples' health service use behavior [5], and the number of patients who attended the hospital during the COVID-19 outbreak was inevitably small compared to previous years. However, our study enrolled patients who had their glycosylated hemoglobin (HbAlc) levels measured during both Periods 1 (November 18 to February 17) and 2 (February 18 to May 17). Newly diagnosed patients with diabetes during Period 1 were diagnosed before COVID-19 outbreak, and newly diagnosed diabetic patients during Period 2 were not included in all cohorts (COVID-19 cohort and non-COVID-19 cohorts). Therefore, the number of newly diagnosed patients with diabetes was not influenced by the COVID-19 outbreak among co- horts and we believe it did not affect our research.

Nevertheless, your opinion is important and should be considered; a rapid decrease of $\mathrm{HbAlc}$ in newly diagnosed patients with diabetes might have affected the results of our study. $\mathrm{HbAlc}$ levels were markedly reduced in groups with baseline above 9.0\%, which was consistent in all cohorts of our study. Peoples with newly diagnosed diabetes, especially those who visit tertiary hospitals, tend to show higher HbAlc levels; considering this, we agree with your opinion that the rapid decrease of $\mathrm{HbAlc}$ might be a consequence of enrollment of newly diagnosed patients with diabetes. However, we found that glycemic control worsened in patients with $\mathrm{HbAlc}<7 \%$ for all cohorts, and the increment of HbAlc levels was significantly higher in the COVID-19 cohort than in the non-COVID-19 cohorts. We believe that lifestyle changes due to social distancing might have caused this distinct increase in HbAlc levels in the COVID-19 cohort. We would like to thank Dr. Noh again for your comprehensive review and advice on our findings. The limitation of our study that you pointed out will be identified through follow-up researches to be conducted later.
Corresponding authors: Keun-Gyu Park (iD https://orcid.org/0000-0002-8403-1298 Division of Endocrinology and Metabolism, Department of Internal Medicine, Kyungpook National University Hospital, School of Medicine, Kyungpook National University, 130 Dongdeok-ro, Jung-gu, Daegu 41944, Korea

E-mail: kpark@knu.ac.kr
This is an Open Access article distributed under the terms of the Creative Commons Attribution Non-Commercial License (https://creativecommons.org/licenses/by-nc/4.0/) which permits unrestricted non-commercial use, distribution, and reproduction in any medium, provided the original work is properly cited. 


\section{CONFLICTS OF INTEREST}

No potential conflict of interest relevant to this article was reported.

\section{REFERENCES}

1. Park SD, Kim SW, Moon JS, Lee YY, Cho NH, Lee JH, et al. Impact of social distancing due to coronavirus disease 2019 on the changes in glycosylated hemoglobin level in people with type 2 diabetes mellitus. Diabetes Metab J 2021;45:109-14.

2. Korean Society of Infectious Diseases; Korean Society of Pediatric Infectious Diseases; Korean Society of Epidemiology; Korean Society for Antimicrobial Therapy; Korean Society for Healthcare-associated Infection Control and Prevention; Ko- rea Centers for Disease Control and Prevention. Report on the epidemiological features of coronavirus disease 2019 (COVID-19) outbreak in the Republic of Korea from January 19 to March 2, 2020. J Korean Med Sci 2020;35:e112.

3. Thu TPB, Ngoc PNH, Hai NM, Tuan LA. Effect of the social distancing measures on the spread of COVID-19 in 10 highly infected countries. Sci Total Environ 2020;742:140430.

4. Park SW, Sun K, Viboud C, Grenfell BT, Dushoff J. Potential role of social distancing in mitigating spread of coronavirus disease, South Korea. Emerg Infect Dis 2020;26:2697-700.

5. Mehrotra A, Chernew M, Linetsky D, Hatch H, Cutler D: The impact of the COVID-19 pandemic on outpatient visits: a rebound emerges. Available from: https://doi.org/10.26099/ds9ejm36 (updated 2020 May 19). 